C-A/AP/\#118 October 2003

\title{
Bunch Patterns and Pressure Rise in RHIC
}

W. Fischer and U. Iriso-Ariz

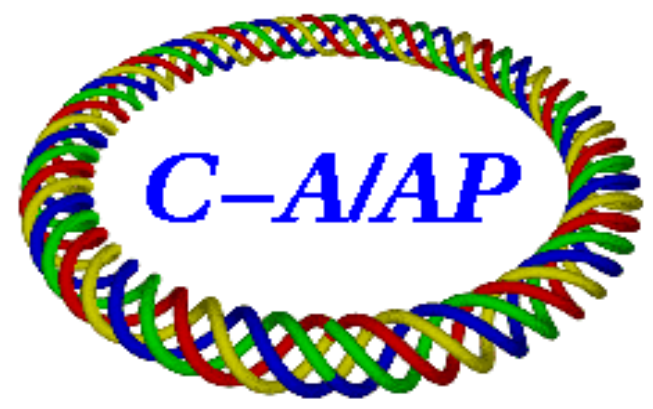

Collider-Accelerator Department Brookhaven National Laboratory

Upton, NY 11973 
BNL C-A/AP/118

\title{
Bunch Patterns and Pressure Rise in RHIC
}

\author{
W. Fischer and U. Iriso-Ariz
}

November 10, 2003

\begin{abstract}
After the upgrade of the RHIC injection system, the bunch number can be increased from currently 56 to 112 . However, with large bunch numbers and high-intensity bunches a significant pressure rise can be observed in several warm locations. This affects the experimental background and beam lifetime. We discuss the optimal distribution of bunches along the circumference for bunch numbers between 56 and 112 .
\end{abstract}

\section{Introduction}

In 2001 first tests were made to double the bunch number in RHIC from 56 to 112 . It became clear that an upgrade of the injection system was needed to reduce the injection kicker timing jitter and drift, and allow for easy drift corrections from the Main Control Room. These upgrades were finished in 2002 [1-3].

However, injection of many high-intensity bunches lead to an unacceptable pressure rise in a number of warm locations [4,5]. After more baking during the 2002 shut down the vacuum performance improved, but a pressure rise was still observable with intense beams $[6,7]$. Also during the shutdown 11 electron detectors were installed in 2 interaction regions. With these electron clouds could be observed concurrently with pressure rises [8].

During the recent deuteron-gold run, RHIC was routinely operated with 110 bunches in both beams from mid January to the end of February 2003. With increasing bunch currents, backgrounds in all experiments became an issue. The Phobos background could be linked to pressure rises $[9,10]$. End of February, the bunch number was reduced again to 55 with clear improvements in the Phobos background. Around the same time $\beta^{*}$ at Phobos was raised from 2 to $4 \mathrm{~m}$, and at Brahms from 2 to $3 \mathrm{~m}$. The Phobos $\beta^{*}$ was later reduced again to $3 \mathrm{~m}$. Also at the same time, a newly commissioned coherence monitor [11] allowed to eliminate transverse instabilities on the ramp.

Although further improvements are made to the vacuum system, it may still not be possible to operate with 112 bunches in the next Run. But it may be possible to run with more than 56 bunches. We discuss how bunch numbers between 56 and 112 should be distributed along the circumference, in particular the case of 68 bunches, which would yield $20 \%$ more luminosity compared to the current bunch number.

In the following we will use triplets of integer numbers $\left(k_{s}, k_{b}, k_{g}\right)$ to describe bunch patterns. $k_{s}$ gives the bunch spacing in buckets, $k_{b}$ the number of bunches filled with that spacing, and $k_{g}$ the number of "phantom" bunches added, i.e. bunches that are not filled 

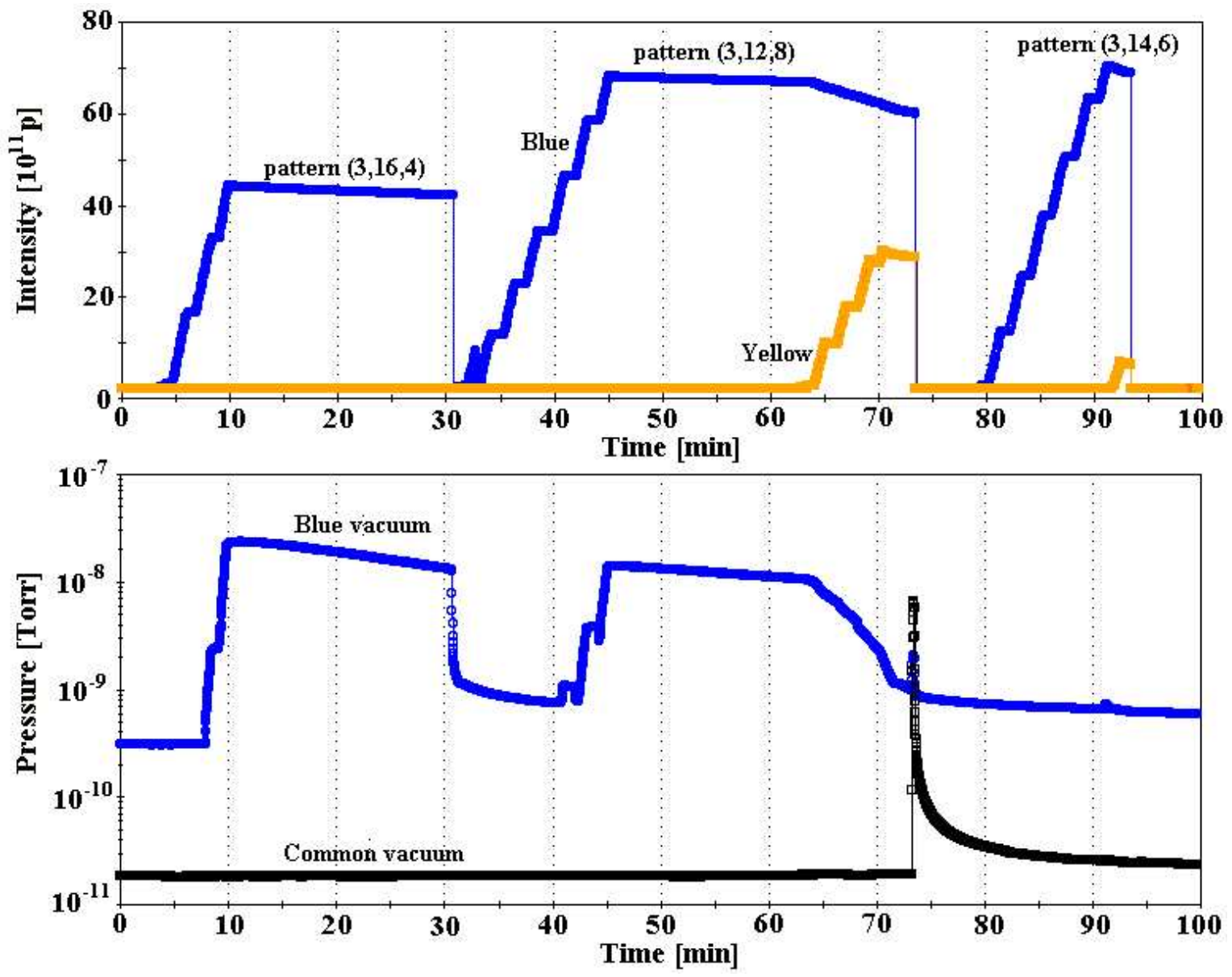

Figure 1: Blue and common vacuum with different intensities and bunch pattern.

in and therefore create a gap. Changing patterns can then be described by adding a new triplet. For example the configuration $(2,2,1)(3,4,0)$ would correspond to the pattern

$$
\text { 1-0-1-0-0-0-1-0-0-1-0-0-1-0-0-1-0-0 }
$$

where 1 denotes a filled and 0 denotes an empty bucket. If not otherwise noted, it is assumed that a pattern repeats until the abort gap is reached.

\section{Experimental Observations}

On 23 April 2003 three different bunch patterns were tested: $(3,16,4),(3,12,8)$ and $(3,14,6)$. In Fig. 1 the total intensity is seen during the fill as well as the pressure in a Blue and a common beam pipe, both of them located in the 2 o'clock interaction region.

In the first fill, with pattern $(3,16,4), 41$ bunches were filled for a total intensity of $44.3 \cdot 10^{11} \mathrm{p}$, or an average bunch intensity of $N_{b}=1.1 \cdot 10^{11}$. After 41 bunches the pressure exceeded $10^{-6}$ Torr in one location in the 2 o'clock interaction region, and electron clouds 

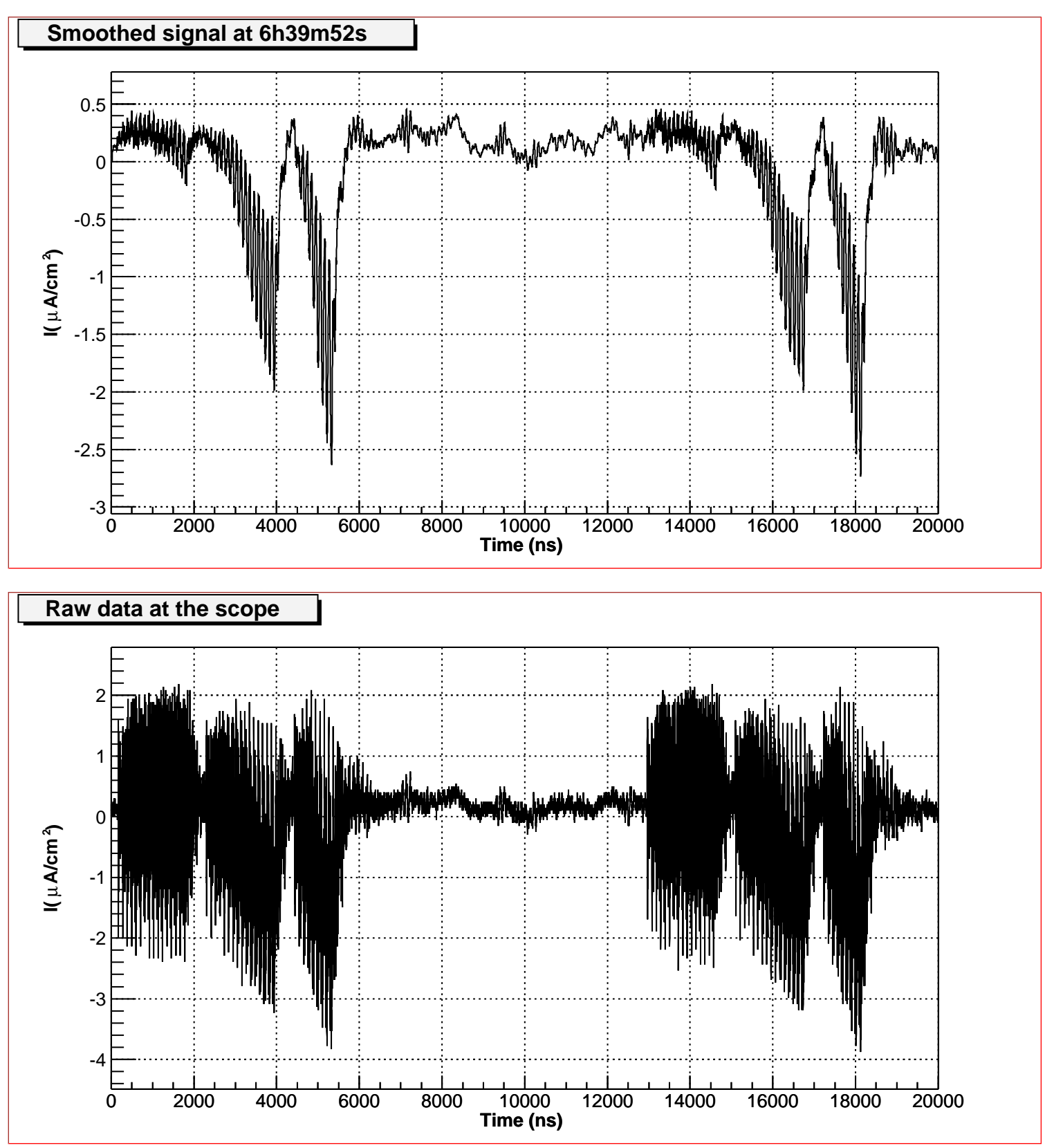

Figure 2: Electron detector signal for the bunch pattern $(3,16,4)$. The raw signal is shown in the lower part, the smoothed signal in the upper part. One turn is $12.8 \mu \mathrm{s}$. 

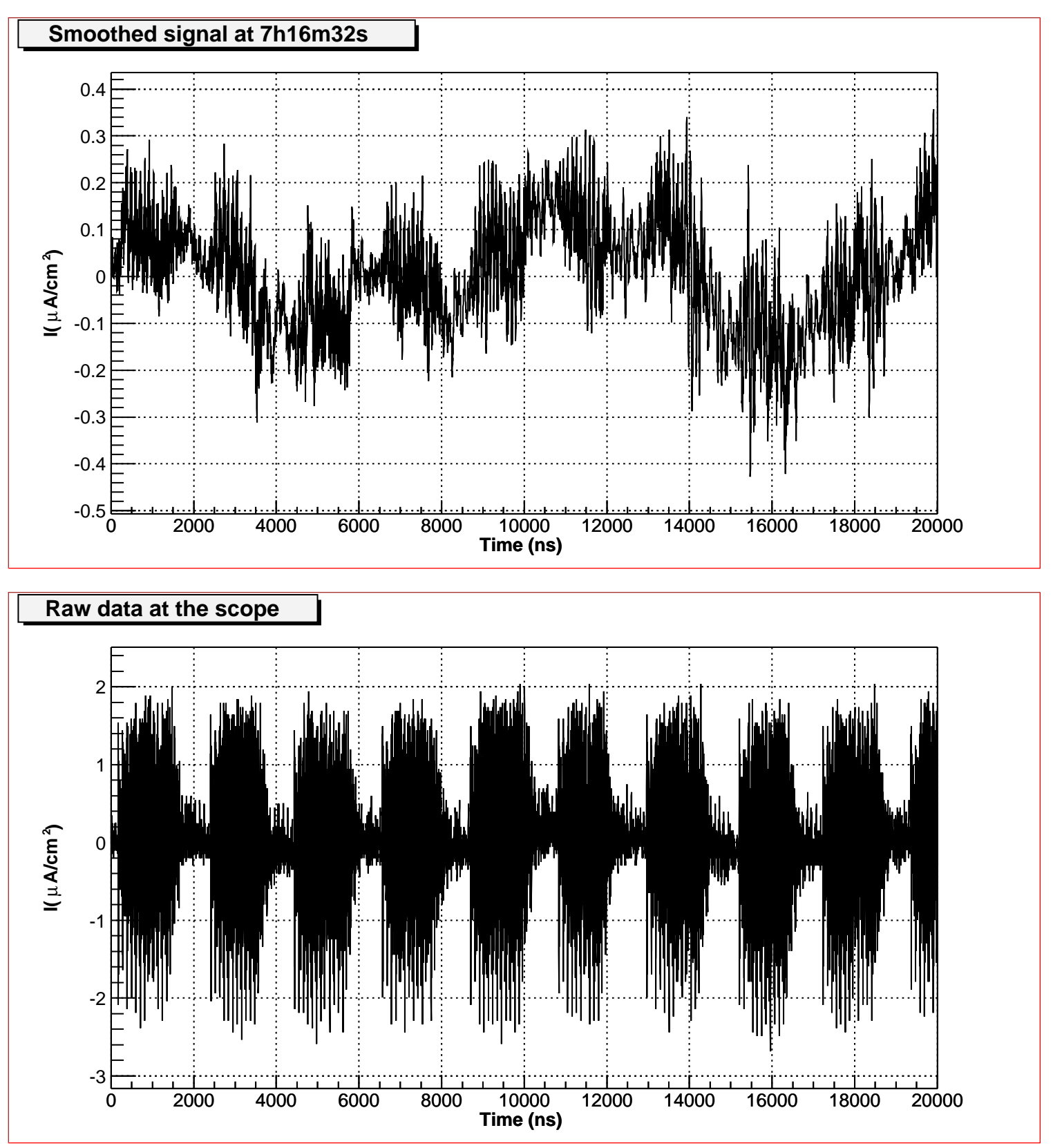

Figure 3: Electron detector signal for the bunch pattern $(3,12,8)$. The raw signal is shown in the lower part, the smoothed signal in the upper part. One turn is $12.8 \mu \mathrm{s}$. 
could be observed. Fig. 2 shows the signal from an electron detector, placed in the Blue 2 o'clock region, for $20 \mu \mathrm{s}$ (a turn in RHIC is $12.8 \mu \mathrm{s}$ ). The raw data (bottom) are very noisy. Using a $20 \mathrm{MHz}$ numerical smoothing filter, the electron signal becomes clear (top).

In the second fill, with pattern $(3,12,8), 69$ bunches were filled for a total intensity of $68.1 \cdot 10^{11} \mathrm{p}$, or an average bunch intensity of $N_{b}=1.0 \cdot 10^{11}$. All bunches could be filled in and the pressure rose to values somewhat smaller than those in the first fill, which had higher bunch but lower total intensity. Electron clouds could signals in this case are less clear (Fig. 3). Even after smoothing (top), the signal is noisy, and about an order of magnitude smaller than for fill 1. After Blue was filled completely, the Yellow ring was filled with the same bunch pattern. The Yellow beam lead to a clear deterioration in the Blue beam lifetime due to insufficient vertical separation in the interaction regions. This lead to beam-beam interactions. These affected some Blue bunches much stronger than others. Only 37 Yellow bunches were filled since the transfer line became unavailable. A total of $30.1 \cdot 10^{11} \mathrm{p}$ were filled into Yellow, with an average bunch intensity of $N_{b}=0.8 \cdot 10^{11}$. Beams are nominally separated vertically in the interaction regions but here the separation was likely to be reduced due to orbit drifts, and the Blue and Yellow beam lifetimes were severely affected by the beam-beam interaction. After the Yellow filling was stopped, it was attempted to ramp both beams. Upon ramp preparation, a loss monitor in the 4 o'clock interaction region aborted the fill. The beam loss lead to a pressure rise in both the Blue and common vacuum.

In a third fill, with pattern $(3,14,6), 78$ bunches were filled for a total intensity of $70.2 \cdot 10^{11} \mathrm{p}$, or an average bunch intensity of $N_{b}=0.9 \cdot 10^{11}$. In this case neither pressure rises nor electron clouds could be observed. The initial low bunch intensity was increased during the fill, leading to differences in the bunch intensity of about a factor 3 between the first and last bunches. As a result of longitudinal injection mismatch, the first bunches filled their respective buckets. The fill of Yellow beam had to be stopped again due to the unavailability of the transfer line, and an attempt to ramp was also aborted due to losses in the 4 o'clock interaction region.

The observations are summarized in Tab. 1. The reference case denotes nominal running conditions, not yet achieved in operation. For each fill a luminosity scaling factor is computed, assuming that both rings can be filled with the respective bunch pattern. We assume that the luminosity scales linear with the bunch number, and quadratic with the bunch intensity (i.e. we operate below the beam-beam limit).

A comparison of the three cases is complicated by the fact that the bunch intensity and bunch length are not the same for all fills. Bunch intensity and length are comparable for fills 1 and 2, which also show similar vacuum behavior. However, fill 2 would yield about $40 \%$ more luminosity. Fill 2 also fares better than fill 3 in terms of luminosity. Fill 3 has bunch lengths that are larger by a factor of 2 compared to fills 1 and 2. This, and the reduced bunch intensity, can account for the suppression of electron clouds.

Note that fill 2 has the shortest bunch trains with the 3 bucket spacing. This may be an indication that more uniform bunch patterns are favorable in terms of vacuum performance. 
Table 1: Comparison of bunch patterns tested in RHIC at injection.

\begin{tabular}{lccccc}
\hline \hline parameter & unit & $\begin{array}{c}\text { reference } \\
\text { case }\end{array}$ & $\begin{array}{c}\text { fill } \\
\text { no 1 }\end{array}$ & $\begin{array}{c}\text { fill } \\
\text { no 2 }\end{array}$ & $\begin{array}{c}\text { fill } \\
\text { no 3 }\end{array}$ \\
\hline bunch pattern & $\ldots$ & $(6,1,0)$ & $(3,16,4)$ & $(3,12,8)$ & $(3,14,6)$ \\
no of bunches & $\ldots$ & 56 & 41 & 69 & 78 \\
average bunch intensity $N_{b}$ & $10^{11} \mathrm{p}$ & 1.0 & 1.1 & 1.0 & 0.9 \\
total intensity & $10^{11} \mathrm{p}$ & 56.0 & 44.3 & 68.1 & 70.2 \\
full bunch length & $\mathrm{ns}$ & $\ldots$ & 16.5 & 17.6 & 34.2 \\
pressure rise & $\ldots$ & $\ldots$ & yes & yes & no \\
luminosity scaling factor & $\ldots$ & 1.00 & 0.88 & 1.23 & 1.13 \\
\hline \hline
\end{tabular}

\section{Electron Cloud Simulations}

Operational experience from Run-3 suggests that a bunch number increase from 56 to 112 is too large a step under the current vacuum conditions. However, a bunch number between 56 and 112 may be accommodated. We chose 68 bunches for the following investigation, which would yield a luminosity increase of $20 \%$. In our simulations, 68 bunches is also at the border of stability with respect to electron clouds. Small reductions in, for example, the secondary emission yield or electron reflectivity at small energies result in complete suppression of electron clouds.

The computer code NCSEC was used to simulate the effect of different bunch patterns on the evolution of electron clouds. NCSEC was written by M. Blaskiewicz, a description of the code can be found in Ref. [12]. To compare the effect of different bunch patterns on the vacuum, we assume the pressure is a monotonic function of the average electron cloud line density $\rho_{\text {ave }}$, i.e. we search for the bunch pattern that minimizes $\rho_{\text {ave }}$. This disregards variations in the electron current into the wall, and variations in the energy spectra of the electrons with the different bunch patterns. However, one can reasonably assume that the current into the wall and the electron energies increase with the electron line density [13].

Five cases were simulated, each with 68 bunches, but in different bunch patterns. The basic parameters used in the simulations are shown in Tab. 2 (see Ref. [5] for a description of these parameters). Assuming $\mathrm{Au}^{79+}$ as particle species, all cases showed electron cloud suppression for $N_{b}=0.8 \cdot 10^{9}$, and sustained electron clouds for $N_{b}=1.0 \cdot 10^{9}$.

In the first case (Fig. 4) all 68 bunches are concentrated at the beginning of a turn, the pattern is $(3,68,52)$. The electron cloud line density saturates within less than half a turn and reaches a maximum of $0.92 \mathrm{nC} / \mathrm{m}$. The average line density is $0.30 \mathrm{nC} / \mathrm{m}$. In the second case (Fig. 5) bunches are places in 3 trains with the pattern $(3,23,17)$. The maximum electron cloud line density is reduced to $0.67 \mathrm{nC} / \mathrm{m}$, and the average to $0.14 \mathrm{nC} / \mathrm{m}$. In the third case (Fig. 6) the bunches are distributed in 6 trains with the pattern $(3,12,8)$. The maximum electron cloud line density reaches only $0.28 \mathrm{nC} / \mathrm{m}$, and the average only $0.10 \mathrm{nC} / \mathrm{m}$ after reaching a stationary state. In the fourth case (Fig. 7), 6 mini-trains with 3 buckets spacing are inserted in a pattern with 6 buckets difference between bunches, for a pattern of $(3,4,0)(6,8,0)$. The maximum line density is again 
Table 2: List of input parameters for electron cloud simulations.

\begin{tabular}{lcc}
\hline \hline parameter & unit & value \\
\hline bunch spacing & $\mathrm{ns}$ & $108 / 216$ \\
beam offset & $\mathrm{mm}$ & 0 \\
bunches & $\ldots$ & 68 \\
\hline rms beam radius & $\mathrm{mm}$ & 2.4 \\
pipe radius & $\mathrm{mm}$ & 60 \\
\hline electrons generated/bunch & $\ldots$ & 35000 \\
electron generation radius & $\mathrm{mm}$ & 60 \\
\hline full bunch length & $\mathrm{ns}$ & 15 \\
bunch shape parameter $n$ & $\ldots$ & 3 \\
bunch charge & $\mathrm{nC}$ & 12.6 \\
\hline longitudinal slices & $\mathrm{per}$ turn & 108000 \\
macro-particles, initially & $\ldots$ & 25 \\
smoothing length $d$ & $\mathrm{~mm}$ & 1.0 \\
\hline$\rho_{\text {ce }}$, initial & $\mathrm{pC} \cdot \mathrm{m}$ & 0.2 \\
$P_{0}[14,15]$ & $\ldots$ & 0.6 \\
$P_{\infty}$ & $\ldots$ & 0.2 \\
$E_{\text {reflect }}$ & $\mathrm{eV}$ & 60 \\
$P_{\text {rediffuse }}$ & $\ldots$ & 0.5 \\
$\delta_{\text {max }}$ & $\ldots$ & 2.1 \\
$E_{\text {max }}$ & $\mathrm{eV}$ & 310 \\
$E_{\text {secondary }}$ & $\mathrm{eV}$ & 8.9 \\
$\alpha_{\delta}$ & $\ldots$ & 1.0 \\
$\alpha_{\theta}$ & & 1.0 \\
\hline \hline
\end{tabular}

reduced, to $0.22 \mathrm{nC} / \mathrm{m}$, while the average changed only little. In the fifth case (Fig. 8), the bunches are distributed in the most uniform way around the circumference, with the pattern $(3,2,0)(6,4,0)$. For this case the maximum and average line densities are reduced again, although only by a small amount compared to case three.

The simulation cases are summarized in Tab. 3. Both the peak line density and the average electron cloud line density are maximized if the bunches are concentrated in a single train of minimum bunch spacing, and minimized if the bunches are uniformly distributed around the circumference. With RHIC's six-fold symmetry, the bunch pattern must also have a three-fold symmetry to have approximately the same number of collision in all experiments. (Due to the abort gap some experiments have about $10 \%$ less bunchbunch collisions than other experiments.) 

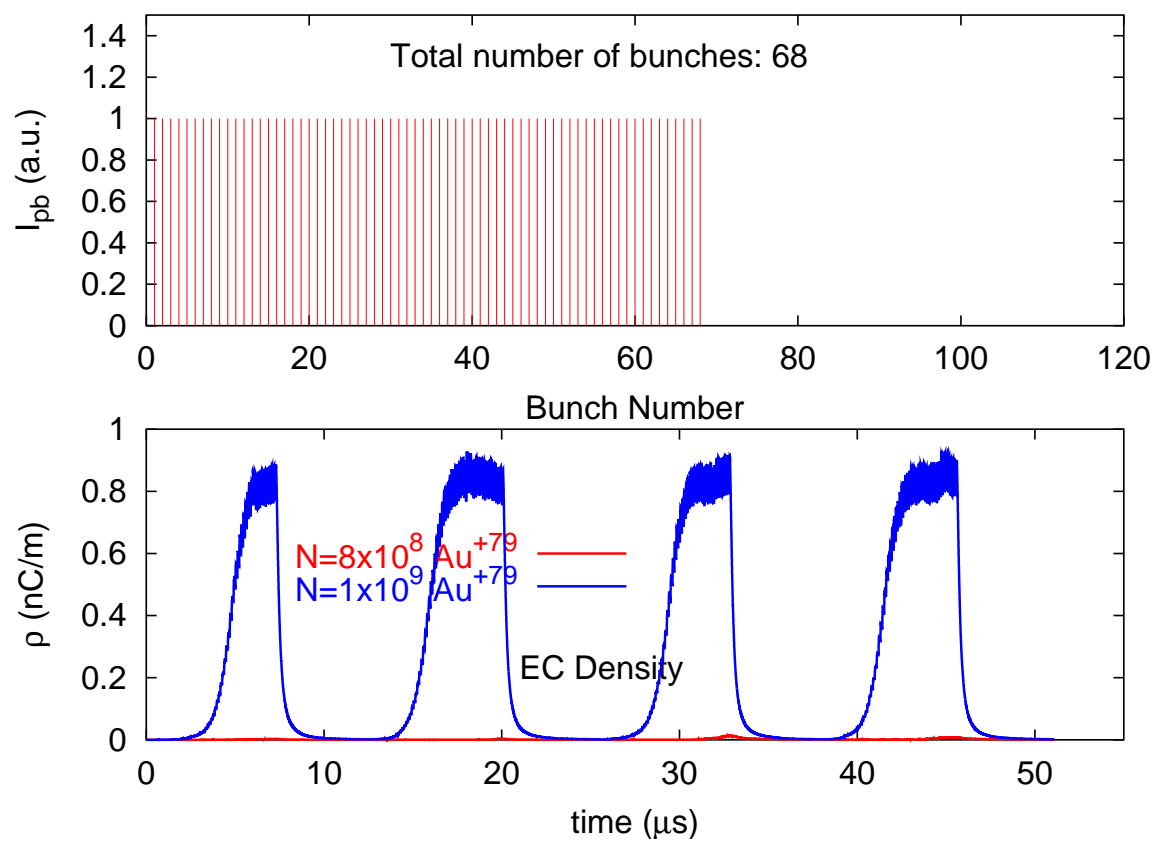

Figure 4: Simulated electron cloud evolution for 68 bunches and 4 turns (lower part) for the pattern $(3,68,52)$ shown in the upper part. Note that the upper and lower part have different time scales.
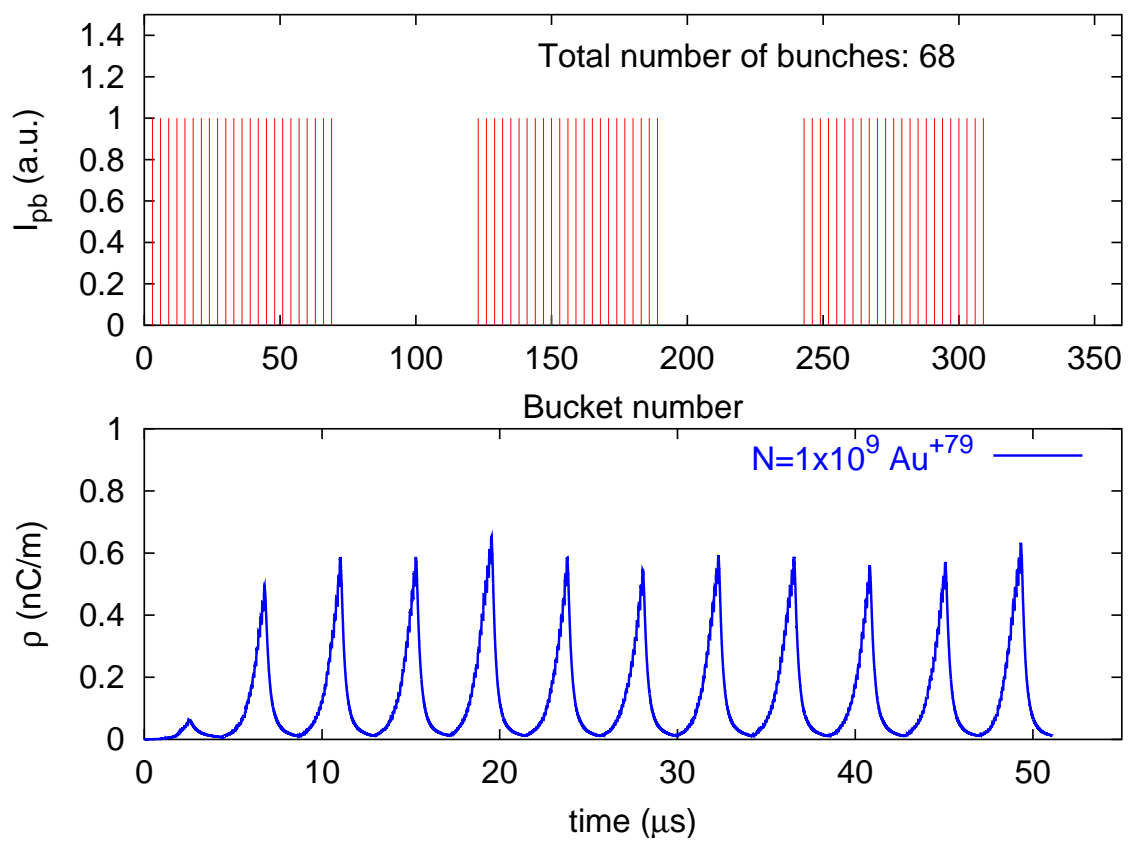

Figure 5: Simulated electron cloud evolution for 68 bunches and 4 turns (lower part) for the pattern $(3,23,17)$ shown in the upper part. Note that the upper and lower part have different time scales. 

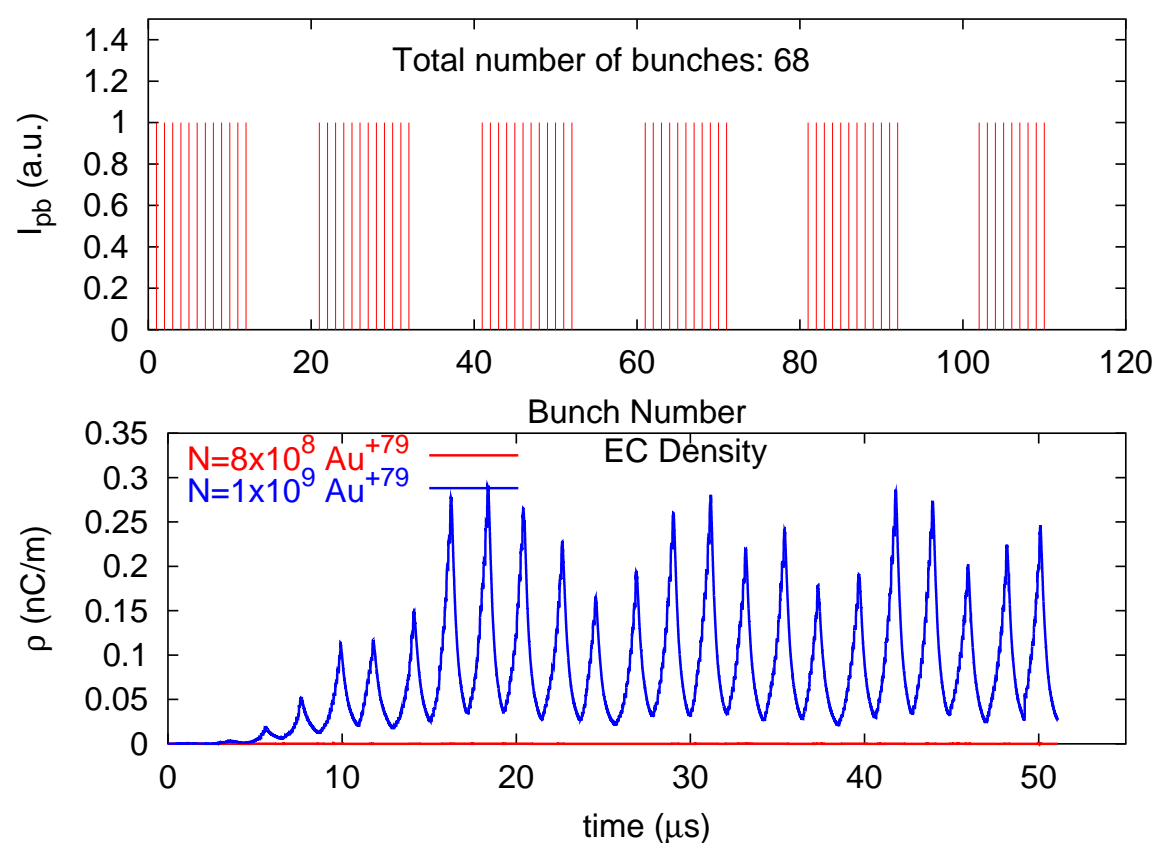

Figure 6: Simulated electron cloud evolution for 68 bunches and 4 turns (lower part) for the pattern $(3,12,8)$ shown in the upper part. Note that the upper and lower part have different time scales.
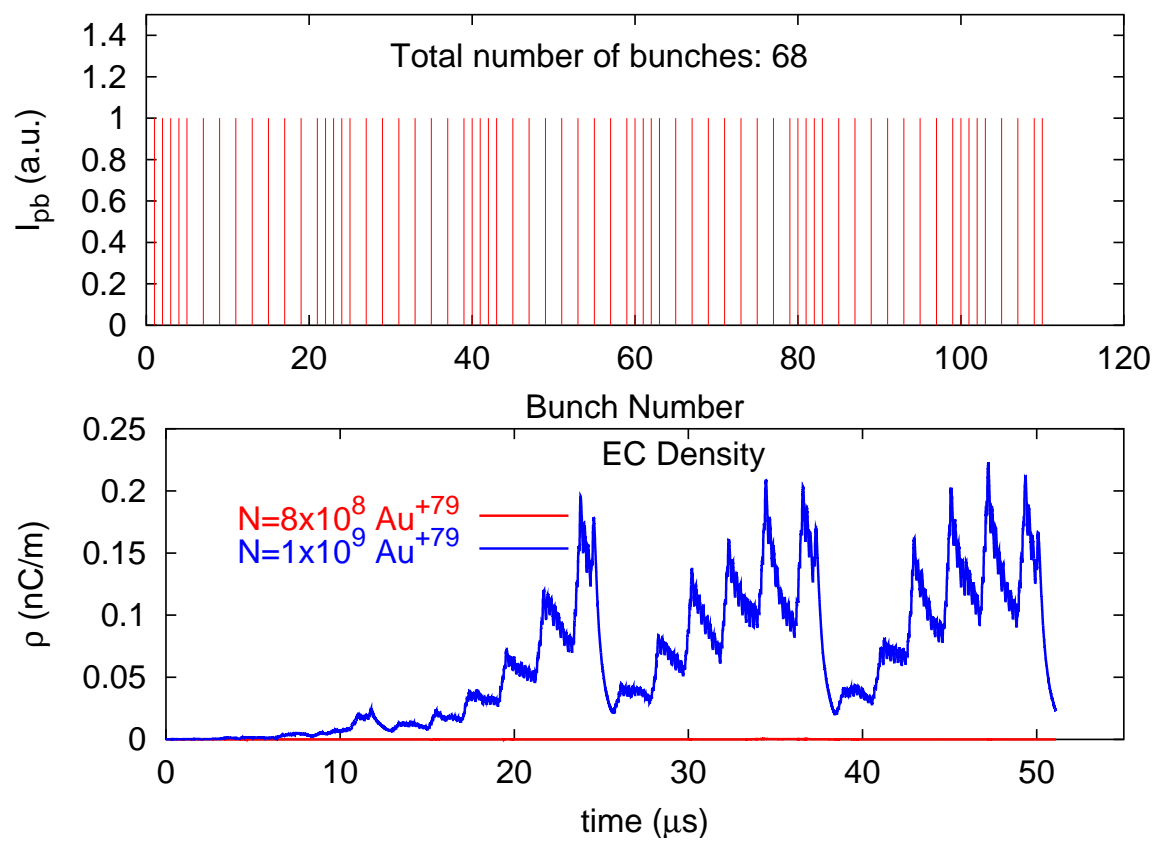

Figure 7: Simulated electron cloud evolution for 68 bunches and 4 turns (lower part) for the pattern $(3,4,0)(6,8,0)$ shown in the upper part. Note that the upper and lower part have different time scales. 

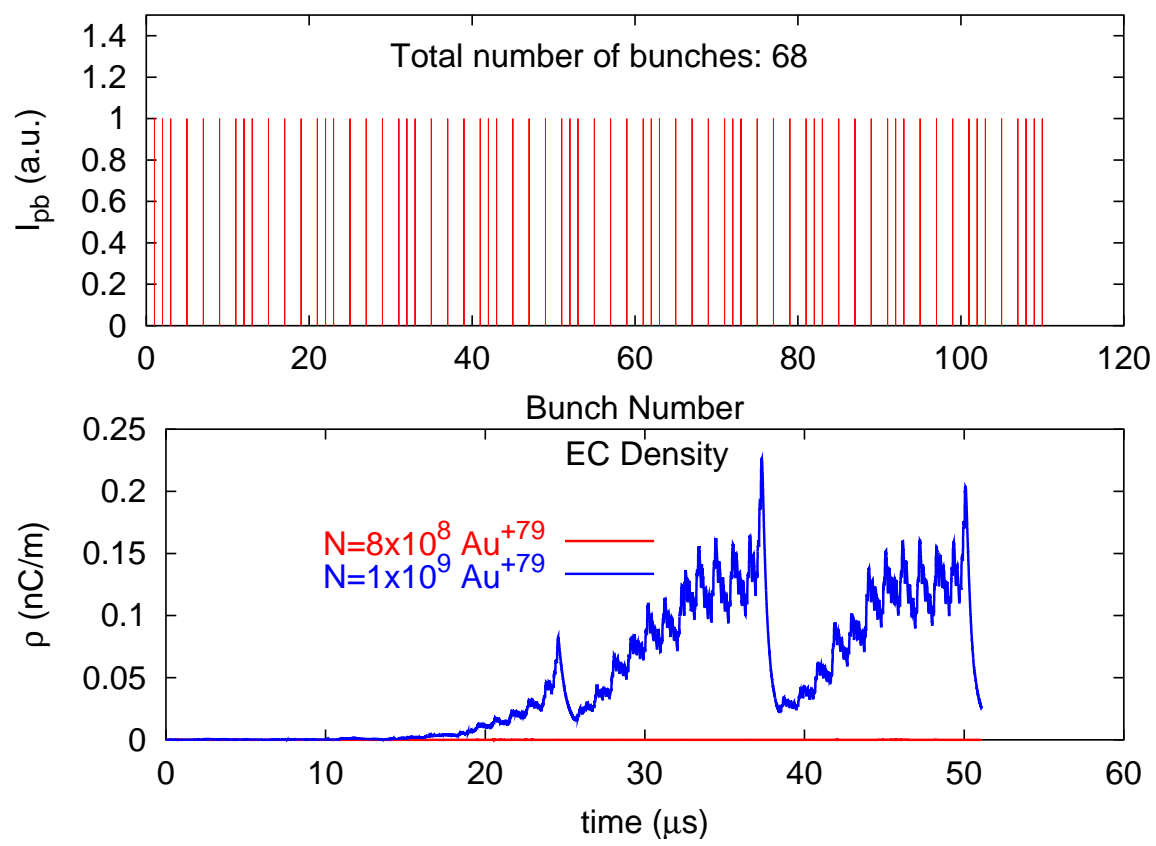

Figure 8: Simulated electron cloud evolution for 68 bunches and 4 turns (lower part) for the pattern $(3,2,0)(6,4,0)$ shown in the upper part. Note that the upper and lower part have different time scales.

Table 3: Comparison of bunch patterns tested in simulations.

\begin{tabular}{lcccccc}
\hline \hline parameter & unit & case & case & case & case & case \\
& & no 1 & no 2 & no 3 & no 4 & no 5 \\
\hline bunch pattern & $\ldots$ & $(3,68,52)$ & $(3,23,17)$ & $(3,12,8)$ & $(3,4,0)(6,8,0)$ & $(3,2,0)(6,4,0)$ \\
no of bunches & $\ldots$ & 68 & 68 & 68 & 68 & 68 \\
bunch intensity $N_{b}$ & $10^{9} \mathrm{Au}$ & 1.0 & 1.0 & 1.0 & 1.0 & 1.0 \\
total intensity & $10^{9} \mathrm{Au}$ & 68.0 & 68.0 & 68.0 & 68.0 & 68.0 \\
maximum line density $\rho_{\max }$ & $\mathrm{nC} / \mathrm{m}$ & 0.92 & 0.67 & 0.28 & 0.22 & 0.20 \\
average line density $\rho_{\text {ave }}$ & $\mathrm{nC} / \mathrm{m}$ & 0.30 & 0.14 & 0.10 & 0.10 & 0.09 \\
\hline \hline
\end{tabular}

\section{Bunch Patterns in B-Factories}

In the quest for higher luminosities, both KEKB and PEP-II have increased bunch number and changed the bunch pattern several times. Electron clouds are created in the positron rings of both machines, and thus their experience may offer some guidance for bunch patterns in RHIC. The bunch patterns in both machines are also influenced by other constraints, most importantly the beam-beam interaction. With short bunch spacings bunches experience a stronger beam-beam interaction since they may have more parasitic crossings in the interaction region [16-20]. Both machines have large a flexibility to create different bunch patters. In Tab. 4 the latest running conditions are summarized. 
Table 4: Recent bunch patterns used in the positron rings of the B-factories [16-20].

\begin{tabular}{lccc}
\hline \hline property & unit & KEKB & PEP-II \\
\hline circumference $C$ & $\mathrm{~m}$ & 3016 & 2200 \\
beam energy $E$ & $\mathrm{GeV}$ & 3.5 & 3.1 \\
harmonic number $h$ & $\ldots$ & 5120 & 3492 \\
bucket spacing $t_{s}$ & $\mathrm{~ns}$ & 2.0 & 2.1 \\
number of bunches $n_{b}$ & $\ldots$ & 1280 & 1034 \\
charge per bunch $N_{b}$ & $10^{11} \mathrm{e}^{+}$ & 0.55 & 0.69 \\
rms bunch length $\sigma_{s}$ & $\mathrm{~mm}$ & 4 & 13 \\
bunch pattern & $\ldots$ & $(3,1,0)(4,1,0)$ & $(3,10,2)(3,11,1)$ \\
\hline \hline
\end{tabular}

KEKB now has settled on a pattern, in which bunches are distributed approximately uniformly, spaced 6 and 8 ns apart, with an average of 7 ns [19]. At PEP-II a number of patterns were tried $[18,20]$. Some cases were found in which the same number of bunches lead to higher luminosity when concentrated in trains.

\section{Bunch Pattern Implementation in the Control System}

In the past, the RHIC control system allowed to chose the bunch spacing and the number of bunches. Patterns with changing bunch spacing could only be created manually by switching off the Booster when a bucket was not to be filled.

A new implementation allows for more flexible bunch patterns. Fig. 9 shows an interface that now allows to arbitrarily select any of the 120 buckets that are spaced 3 buckets apart, with the exception of the abort gap. The minimum spacing of 3 buckets is given by the injection kicker rise time.

Currently, not all possible patterns selected in this way can be implemented in operation. The current hardware requires that the distance between bunches, measured in buckets, is a number that leaves no rest when 360 is divided by it [21]. Hardware modifications are needed to overcome this restriction. The additional bunch patterns, available without the current limitations, would have little impact on the machine operation.

\section{Summary}

We analyzed the effect of different bunch patterns on the vacuum and luminosity experimentally and in simulations, assuming electron clouds as the main source for pressure rises. To maximize the luminosity, the bunch intensity should be increased first to the maximum possible. Then, more bunches can be injected to further increase the luminosity. Our simulations show that bunch patterns with the most uniform distributions of bunches along the circumference minimize the pressure rise. This conclusion is supported by the available experimental data. Due to RHIC's 6-fold symmetry, the bunch pattern 


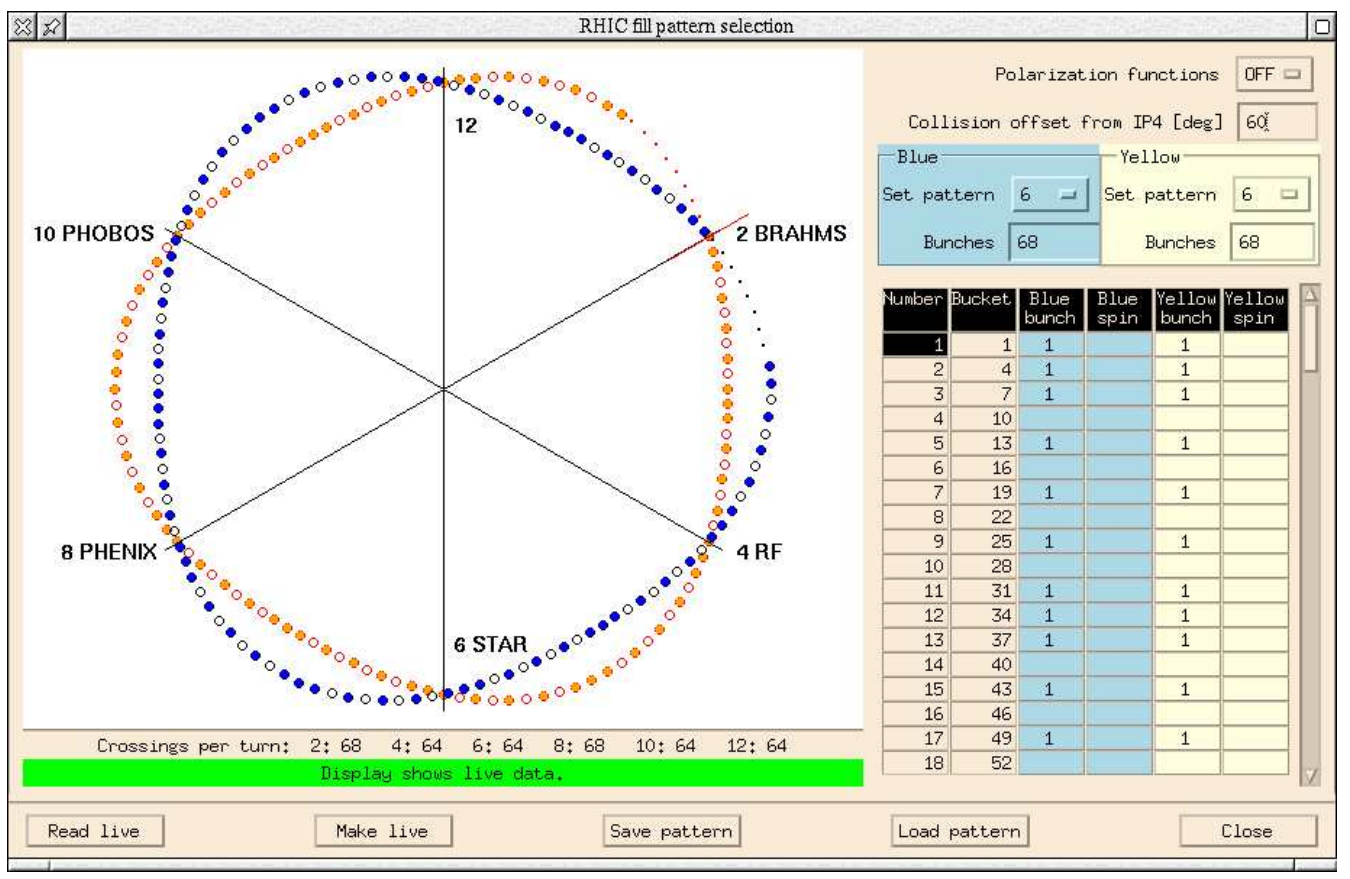

Figure 9: Interface to set the bunch pattern. 112 possible locations for bunches can be arbitrarily filled or not filled with a bunch. The picture shows settings for the pattern $(3,2,0)(6,4,0)$, where bunch 1 of the Blue beam collides with bunch 1 of the Yellow in 2 o'clock and 8 o'clock.

must also have a 3-fold symmetry to provide approximately the same number of collisions to all experiments.

\section{Acknowledgements}

The authors are thankful for discussions with M. Blaskiewicz, F.-J. Decker, M. Furman, H.C. Hseuh, K. Ohmi, S. Peggs, T. Satogata, P. Thieberger and S.Y. Zhang.

\section{References}

[1] H. Hahn, W. Fischer, and J. Tuozzolo, "All-Ferrite RHIC Injection Kickers", proceedings of the 2001 Particle Accelerator Conference, Chicago, BNL-67983 (2001).

[2] H. Hahn, W. Fischer, and Y.K. Semertzidis, "Improvements of the RHIC Injection Kicker System", BNL C-A/AP/74 (2002).

[3] H. Hahn, W. Fischer, Y.K. Semertzidis, and D.S. Warburton, "Up-graded RHIC Injection Kicker System", proceedings of the 2003 Particle Accelerator Conference, Portland, Oregon (2003).

[4] S.Y. Zhang, "RHIC Vacuum Pressure Bump", BNL C-A/AP/67 (2002). 
[5] W. Fischer, M. Bai, J.M. Brennan, M. Blaskiewicz, P. Cameron, H.C. Hseuh, H. Huang, W. MacKay, T. Roser, T. Satogata, L.A. Smart, D. Trbojevic, and S.Y. Zhang, "Vacuum Pressure Rise with Intense Ion Beams in RHIC", proceedings of the 2002 European Particle Accelerator Conference, Paris, BNL-68937 (2002).

[6] H. Hseuh, P. He, R.C. Lee, M. Mapes, L. Smart, D. Weiss, S.Y. Zhang, "Improvement of RHIC Warm Beam Vacuum for High Intensity Operation", proceedings of the 2003 Particle Accelerator Conference, Portland, Oregon (2003).

[7] S.Y. Zhang, M. Bai, M. Blaskiewicz, P. Cameron, A. Drees, W. Fischer, D. Gassner, J. Gullotta, P. He, H.C. Hseuh, H. Huang, U. Iriso-Ariz, R. Lee, W.W. MacKay, B. Oerter, V. Ptitsyn, V. Ponnaiyan, T. Roser, T. Satogata, L. Smart, D. Trbojevic, and K. Zeno, "RHIC Pressure Rise and Electron-Cloud", proceedings of the 2003 Particle Accelerator Conference, Portland, Oregon (2003).

[8] U. Iriso-Ariz, M. Blaskiewicz, A. Drees, W. Fischer, S. Peggs, and D. Trbojevic, "Electron Cloud and Pressure Rise Simulations for RHIC", proceedings of the 2003 Particle Accelerator Conference, Portland, Oregon (2003).

[9] U. Iriso-Ariz, "10 O'Clock Pressure Rise", RHIC Retreat 2003 presentation (2003).

[10] S.Y. Zhang, "Experiment Background in RHIC Deuteron-Gold Run", BNL C-A/AP/107 (2003).

[11] R. Michnoff, private communication (2003).

[12] W. Fischer, J.M. Brennan, M. Blaskiewicz, and T. Satogata, "Electron Cloud Measurements and Simulations for the Brookhaven Relativistic Heavy Ion Collider", Phys. Rev. ST Accel. Beams 5, 124401 (2002).

[13] M. Blaskiewicz, private communication (2000).

[14] M.A. Furman and M. Pivi, "Microscopic probabilistic model for the simulation of secondary electron emission", LBNL-49711, CBP Note-415 (2002).

[15] B. Henrist, N. Hilleret, M. Jimenez, C. Scheuerlein, M. Taborelli, G. Vorlaufer, "Secondary Electron Data for the Simulation of Electron Cloud", proceedings of the ECLOUD'02 Workshop at CERN, CERN Yellow Report CERN-2002-001 (2002).

[16] K. Ohmi, M. Tawada, and Y. Funakoshi, "Collision with finite crossing angle at KEKB", proceedings of the workshop Beam-Beam'03, Montauk, New York, AIP conference proceedings, to be published (2003).

[17] W. Kozanecki, Y. Cai, F.-J. Decker, R. Holtzapple, J. Seeman, M. Sullivan, U. Wienands, "Beam-Beam Performance of the SLAC B-Factory", proceedings of the workshop Beam-Beam'03, Montauk, New York, AIP conference proceedings, to be published (2003). 
[18] F.-J. Decker, A. Kulikov, M. Sullivan, "Bunch Pattern By-3 in PEP-II", proceedings of the 2003 PAC, Portland, Oregon (2003).

[19] K. Ohmi, private communication (2003).

[20] F.-J. Decker, private communication (2003).

[21] J.M. Brennen, J. Delong, T. Hayes, T. Le, L. Hoff, and F. Severion private communication (2003). 